\title{
Grundy Functions and Linear Games
}

\author{
By
}

\author{
Masahiko SATo*
}

As is well known, the idea of Grundy functions and Grundy's theorem are very important and useful when we consider the Cartesian product of games. Of course, there are several proofs for Grundy's theorem. ${ }^{1)}$ Yet, we think, these proofs do not answer well the question why the binary sum operation (bitwise addition without carry) must appear in the theorem. An answer for it will be given in this paper.

Mathematically, a game is nothing but a binary relation on a set. Accordingly, its mathematical structure can not be so rich. In $\S 3$, we shall introduce the notion of linear games with richer structures. And we shall prove that any game $G$ is embeddable into a linear game $L(G)$, and that the Grundy function on $L(G)$ is a linear map from $L(G)$ to $N_{.}{ }^{2}$ ) This will easily lead us to a proof of Grundy's theorem.

In $\S 1$, as a preparation for the following $\S \S$, we shall view basic properties of games. In $\S 2$, we shall introduce the notion of compatibility, and extend it to the notion of semicompatibility. Using the notion of semicompatibility, we shall give a characterization of Grundy functions. This, we think, will clarify the meaning of Grundy functions. In $\S 4$, we shall extend the results in $\S 3$, and show that the Grundy function on any linear game is a linear map.

The author wishes to thank Prof. S. Hitotumatu whose literature ${ }^{3)}$ in Japanese guided him to this field. Profs. S. Hitotumatu, A. Nozaki, and

Received October 1, 1971.

Communicated by S. Hitotumatu.

* Department of Mathematics, University of Tokyo, Hongo, Tokyo, 113, Japan.

1) See e.g. [1], [2], [4].

2) By $N$, we mean the set of all non-negative integers.

3) See $[5]$. 
T. Hosoi kindly discussed with the author while he was preparing this paper. Thanks are also due to them.

\section{§1. Preliminaries}

We begin with the definition of games.

Definition 1.1. A game $G$ is a pair $(S, \mu)$, where $S$ is a nonempty set, and $\mu$ is a mapping from $S$ to $2^{S}$.

Thus defined game is a two-person game played alternately by two players, say, A and B. An element $s$ of $S$ is called a state of the game. A state $s$ with $\mu(s)=\varnothing$ is called terminal. The game is played as follows:

If the state is now $s$ and if it is A's turn, A chooses a state $t$ in $\mu(s)$, then it's B's turn and B chooses a state $u$ in $\mu(t)$, and so on. And if it's A (or B)'s turn to change $s$ and if $s$ is terminal, then B (or A) wins the game. That is, whoever terminates the game wins.

Example: A game called one-pile nim is defined as follows:

(1) $S=\mathbf{N}$. (2) $\mu(s)=\{t \in \mathbf{N} \mid t<s\}$.

We denote this game by $N_{1}$.

In what follows, we further assume the following three conditions for any game.

(1) $G$ is progressively finite, that is, for any $s \in S$ there exists some $n \in \boldsymbol{N}$ such that $\mu^{n}(s)=\emptyset .^{4)}$

(2) $G$ is $\mu$-finite, that is, for any $s \in S, \mu(s)$ is a finite set.

(3) $S$ is at most countable.

Later we shall construct various games from given games. It is easy to verify the above three conditions for all such constructed games, so we omit the proofs.

The following lemma is not difficult to show.

Lemma 1.2. For any $G$, there is a one to one mapping $d: S \rightarrow \mathbf{N}$,

4) Note that $\mu^{0}(s)=\{s\}$ and $\mu^{k+1}(s)=\mu\left(\mu^{k}(s)\right)$. 
such that for any $s \in S$ and $t \in \mu(s), d(t)<d(s)$.

We shall fix a mapping $d$ in Lemma 1.2, then $S$ can be well-ordered by using this $d$. We denote the least element of $S$ by $s^{0}$, and the second least element by $s^{1}$, and so on.

Definition 1.3. Let $s$ be a state of a game $G$. We define the length $l(s)$ of $s$ to be the greatest integer $i$ such that $\mu^{i}(s) \neq \emptyset$.

Definition 1.4. A state $s$ is safe if the previous player can force to win, and is unsafe if the next player can force to win.

We denote the whole set of safe states by $P(G)$ (or $P$ if $G$ is understood), and that of unsafe states by $N(G)$ (or $N$ ). Then it is easy to see that

$$
\begin{aligned}
& S=P \cup N, \\
& P \cap N=\varnothing, \\
& P=\bigcup_{i=0}^{\infty} P_{i}, \text { and } \\
& N=\bigcup_{i=0}^{\infty} N_{i},
\end{aligned}
$$

where $P_{i}$ and $N_{i}$ are defined inductively as follows:

$$
\begin{aligned}
& P_{0}=\{s \mid \mu(s)=\emptyset\}, \\
& N_{i}=\left\{s \mid \mu(s) \cap P_{i} \neq \varnothing\right\}, \\
& P_{i+1}=\left\{s \mid \mu(s) \subset N_{i}\right\} .
\end{aligned}
$$

The following lemma is also straightforward.

Lemma 1.5. $s \in P$ iff $\mu(s) \subset N$.

$$
s \in N \text { iff } \mu(s) \cap P \neq \emptyset .
$$

Definition 1.6. Let $G=(S, \mu)$ and $G^{\prime}=\left(S^{\prime}, \mu^{\prime}\right)$ be two games. A mapping $f: S \rightarrow S^{\prime}$ is a homomorphism from $G$ to $G^{\prime}$ if, for any $s \in S$, 
$\mu^{\prime}(f(s))=f(\mu(s))$.

The following theorem shows an essential property of homomorphism.

Theorem 1.7. Let $f: S \rightarrow S^{\prime}$ be a homomorphism from $G$ to $G^{\prime}$. Then $f(P(G)) \subset P\left(G^{\prime}\right)$, and $f(N(G)) \subset N\left(G^{\prime}\right)$. If $f$ is surjective, then $f(P(G))=P\left(G^{\prime}\right)$, and $f(N(G))=N\left(G^{\prime}\right)$.

Proof. We show, by the induction, the following statements for every $i \in \boldsymbol{N}$.

(I $)_{i} \quad s \in P_{i} \Rightarrow f(s) \in P_{i}^{\prime}$.

(II) $)_{i} \quad s \in N_{i} \Rightarrow f(s) \in N_{i}^{\prime}$.

First, we prove $(\mathrm{I})_{0}: s \in P_{0} \Rightarrow \mu(s)=\emptyset \Rightarrow f(\mu(s))=\varnothing$

$\Rightarrow \mu^{\prime}(f(s))=\emptyset \Rightarrow f(s) \in P_{0}^{\prime}$.

We prove (II) ${ }_{\imath}$ supposing (I) $)_{2}: s \in N_{i} \Rightarrow \mu(s) \cap P_{i} \neq \emptyset$

$\Rightarrow f\left(\mu(s) \cap P_{i}\right) \neq \emptyset \Rightarrow f(\mu(s)) \cap f\left(P_{i}\right) \neq \emptyset \Rightarrow \mu^{\prime}(f(s)) \cap P_{i}^{\prime} \neq \emptyset \Rightarrow f(s) \in N_{i}^{\prime}$.

We derive $(\mathrm{I})_{i+1}$ from (II) $)_{i}: s \in P_{i+1} \Rightarrow \mu(s) \subset N_{i}$

$\Rightarrow \mu^{\prime}(f(s))=f(\mu(s)) \subset N_{i}^{\prime} \Rightarrow f(s) \in P_{i+1}^{\prime}$.

The first half of the theorem is thus proved. The latter half is now trivial.

\section{§2. Characterization of Grundy Functions}

Definition 2.1. An equivalence relation $\sim$ on $S$ is compatible with $\mu$, if $s \sim s^{\prime}$ implies that for any $t \in \mu(s)$ there is some $t^{\prime} \in \mu\left(s^{\prime}\right)$ such that $t \sim t^{\prime}$. We call this relation a compatible relation on $G$.

Given an equivalence relation $\sim$ compatible with $\mu$, we can define a quotient game $G / \sim=\left(S^{\prime}, \mu^{\prime}\right)$ as follows:

(1) $S^{\prime}=S / \sim$. (2) Let $[s]$ denote the equivalence class of $s$. We set $\mu^{\prime}([s])=[\mu(s)]$.

Then it is clear that the canonical mapping []$: S \rightarrow S^{\prime}$ is an onto homomorphism from $G$ to $G / \sim$. Next theorem also shows a close connection between homomorphism and compatible relation on $G$. The proof is immediate. 
Theorem 2.2. If $f: G \rightarrow G^{\prime}$ is an onto homomorphism then there exists a compatible relation $\sim$ on $G$ such that $G^{\prime}$ is isomorphic to $G / \sim$.

Definition 2.3. An equivalence relation $\equiv$ on $S$ is called semicompatible with $\mu$, if $s \equiv t$ implies that for any $s^{\prime} \in \mu(s)$ there is either some $t^{\prime} \in \mu(t)$ such that $s^{\prime} \equiv t^{\prime}$ or some $s^{\prime \prime} \in \mu\left(s^{\prime}\right)$ such that $s^{\prime \prime} \equiv s$. We call this relation a semicompatible relation on $G$.

It is well-known that the Grundy function $g_{G}$ (or $g$ when $G$ is understood) on $G$ can be defined recursively by the equation:

$$
g(s)=\min (\boldsymbol{N}-g(\mu(s))) .
$$

Lemma 2.4. Let $\equiv$ be a semicompatible relation on $G$. Then $s \equiv t$ implies $g(s)=g(t)$.

Proof. Suppose the lemma holds for $l(s), l(t)<m$. Suppose now $l(s)=m$ or $l(t)=m$. We may assume that $m=l(s) \geqq l(t)$ and that if $l(u)$ $<l(t)$ then $u \neq t$. Then, by the induction hypothesis, $g(\mu(t)) \subset g(\mu(s))$. Hence $g(s) \geqq g(t)$. But, if $g(s)>g(t)$ then there is some $s^{\prime} \in \mu(s)$ such that $g\left(s^{\prime}\right)=g(t)$. Then, by the induction hypothesis, there are no $t^{\prime} \in \mu(t)$ such that $s^{\prime} \equiv t^{\prime}$. Hence there is some $s^{\prime \prime} \in \mu\left(s^{\prime}\right)$ such that $s^{\prime \prime} \equiv s \equiv t$. By the assumption on $t$ we know $l(t) \leqq l\left(s^{\prime \prime}\right)<l(s)=m$. So, by the induction hypothesis, $g\left(s^{\prime \prime}\right)=g(t)=g\left(s^{\prime}\right)$. This is a contradiction. Thus we have $g(s)=g(t)$.

Since $s$ is safe iff $g(s)=0$, we have

Corollary 2.5. Let $\equiv$ be a semicompatible relation on $G$. If $s \equiv t$ then $s$ is safe iff $t$ is safe.

Definition 2.6. For a semicompatible relation $\equiv$ on $G$, we define $\mu_{\equiv}: S \rightarrow 2^{S}$ as follows:

$$
\mu_{\equiv}(s)=\left\{s^{\prime} \in \mu(s) \mid \forall t \equiv s, \exists t^{\prime} \in \mu(t) \text { such that } t^{\prime} \equiv s^{\prime}\right\}
$$

Lemma 2.7. $\mu_{\equiv}(s)=\left\{s^{\prime} \in \mu(s) \mid \forall t \equiv s, \exists t^{\prime} \in \mu_{\equiv}(t)\right.$ such that $\left.t^{\prime} \equiv s^{\prime}\right\}$. 
Proof. Since $\mu_{\equiv}(t) \subset \mu(t)$, it is clear that the left side of the equation includes the right side. Suppose $s^{\prime} \in \mu_{\equiv}(s)$. Suppose further $t \equiv s$. Then there is some $t^{\prime} \in \mu(t)$ such that $t^{\prime} \equiv s^{\prime}$. We have only to show that $t^{\prime} \in \mu_{\equiv}(t)$. Take any $u \equiv t$, then $u \equiv s$. Since $s^{\prime} \in \mu_{\equiv}(s)$ there is some $u^{\prime} \in \mu(u)$ such that $u^{\prime} \equiv s^{\prime}$. Since $t^{\prime} \equiv s^{\prime}$, this means that for any $u \equiv t$ there is some $u^{\prime} \in \mu(u)$ such that $u^{\prime} \equiv t^{\prime}$. Hence, by the definition of $\mu_{\equiv}(t), t^{\prime} \in \mu_{\equiv}(t)$.

Lemma 2.8. If $\mu(s) \cap P \neq \emptyset$, then $\mu_{\equiv}(s) \cap P \neq \emptyset$.

Proof. Let $t$ be the least element in $S$ such that $s \equiv t$. Clearly, $\mu_{\equiv}(t)=\mu(t)$. Therefore, by Lemma 2.4, $\mu_{\equiv}(t) \cap P \neq \emptyset$. If we take $t^{\prime} \in$ $\mu_{\equiv}(t) \cap P$, then, by Lemma 2.7 , there is some $s^{\prime} \in \mu_{\equiv}(s)$ such that $s^{\prime} \equiv t^{\prime}$. Since $t^{\prime}$ is in $P$, this $s^{\prime}$ is also in $P$.

Corollary 2.9. Let $G_{\equiv}$ be $\left(S, \mu_{\equiv}\right)$. Then $P(G)=P\left(G_{\equiv}\right)$ and $N(G)=$ $N\left(G_{\equiv}\right)$.

If $\equiv$ is an equivalence relation on $S$ semicompatible with $\mu$, then, by Lemma $2.7, \equiv$ is compatible with $\mu_{\equiv}$. Therefore we can define a game $G / \equiv$ by $G / \equiv=G_{\equiv} / \equiv$.

Definition 2.10. Given two games $G$ and $G^{\prime}$, we say a mapping $f$ : $S \rightarrow S^{\prime}$ is a semihomomorphism if $f$ is a homomorphism from $G_{\equiv}$ to $G^{\prime}$ for some semicompatible relation $\equiv$ on $G$.

Since any relation on $S$ is a subset of $S \times S$, the set of all semicompatible relations on $G$ can be ordered by the set inclusion $C$. Let us define an equivalence relation $\equiv_{g}$ on $S$ by the condition that $s \equiv_{g} t$ iff $g(s)=g(t)$. It is easy to see that $\equiv_{g}$ is a semicompatible relation on $G$. By Lemma 2.4, we have the following theorem which characterizes Grundy functions.

Theorem 2.11. (1) Any game $G$ has the maximum semicompatible relation $\equiv_{g}$.

(2) Let [] be the canonical mapping from $G$ to $G / \equiv_{g}$. Then $g(s)=$ 
$l([s])$.

Remark. $G / \equiv_{g}$ is embeddable into one-pile $\operatorname{nim} N_{1}$.

In [3], Grundy and Smith defined $E$ function on any game. By using this function, we can easily show the following two lemmas analogous to Lemma 2.4 and Theorem 2.11.

Lemma 2.12. Let $\sim$ be a compatible relation on $G$. Then $s \sim t$ implies $E(s)=E(t)$.

Lemma 2.13. (1) Any game $G$ has the maximum compatible relation $\sim_{E}$

(2) Let [] be the canonical mapping from $G$ lo $G / \sim_{E}$. Then $E(s)=E([s])$.

Thus we obtain, for any game $G$, the following commutative diagram, where we write $E$ for the game of $E$ values defined in [3].

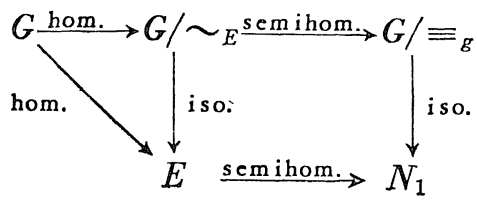

\section{§3. Grundy's Theorem}

Definition 3.1. Let $H$ be a subset of $\operatorname{Map}(S, S) .{ }^{5)}$ For any $s \in S$, we write $H(s)$ for $\{h \in H \mid h(s) \neq s\}$. We define $\mu_{H}: S \rightarrow 2^{S}$ by $\mu_{H}(s)=$ $\{h(s) \mid h \in H(s)\}$.

Definition 3.2. A game $G=(S, \mu)$ is called a linear game, if it satisfies the following conditions.

(I) $S$ is a vector space over $\mathbb{Z}_{2}{ }^{6)}$ whose addition is denoted by $\oplus$.

(II) $\mu=\mu_{H}$ for some $H$ such that

5) Map $(S, S)$ means the set of all the mappings from $S$ to $S$.

6) $Z_{2}$ is the field consisting of two elements 0 and 1. 
(a) $H(s \oplus t)=H(s) \oplus H(t)^{7)}$ for any $s, t \in S$, and

(b) $h(s \oplus t)=h(s) \oplus h(t) \quad$ for any $h \in H$ and $s, t \in S$.

For any game $G=(S, \mu)$ we shall define a linear game $L(G)$. First we define a game $\tilde{G}=(\tilde{S}, \tilde{\mu})$ as follows:

(1) $\tilde{S}$ is a subset of $\prod_{s \in S} \mathbb{N}$ such that $\left(n_{s}\right)_{s \in S} \in \tilde{S}$ iff $n_{s}=0$ for all but finite $s \in S$.

(2) $\left(m_{s}\right)_{s \in S} \in \tilde{\mu}\left(\left(n_{s}\right)_{s \in S}\right)$ iff there are some $s$ and $t \in \mu(s)$ such that $m_{s}=n_{s}-1, m_{t}=n_{t}+1$, and $m_{u}=n_{u}($ if $u \neq s, t)$.

Lemma 3.3. (1) If $\left(n_{s}\right)_{s \in S} \in P(\tilde{G})$ and $\quad\left(m_{s}\right)_{s \in S} \in P(\tilde{G})$ then $\left(n_{s}+m_{s}\right)_{s \in S} \in P(\tilde{G})$.

(2) If $\left(n_{s}\right)_{s \in S} \in P(\tilde{G})$ and $\left(m_{s}\right)_{s \in S} \in N(\tilde{G})$ then $\left(n_{s}+m_{s}\right)_{s \in S} \in N(\tilde{G})$.

Proof. Suppose the lemma holds for $l\left(\left(n_{s}+m_{s}\right)_{s \in S}\right)<k$. Suppose $l\left(\left(n_{s}+m_{s}\right)_{s \in S}\right)=k$.

(1) Take any $\left(l_{s}\right)_{s \in S} \in \tilde{\mu}\left(\left(n_{s}+m_{s}\right)_{s \in S}\right)$. Then, for some $s, l_{s}=$ $n_{s}+m_{s}-1$. We may assume $m_{s}>0$. Then it is easy to see that $\left(l_{s}\right)_{s \in S}=\left(n_{s}+m_{s}^{\prime}\right)_{s \in S}$, for some $\left(m_{s}^{\prime}\right)_{s \in S} \in \tilde{\mu}\left(\left(m_{s}\right)_{s \in S}\right) \subset N(\tilde{G})$. By the induction hypothesis, $\left(l_{s}\right)_{s \in S} \in N(\tilde{G})$. Hence $\left(n_{s}+m_{s}\right)_{s \in S} \in P(\tilde{G})$.

(2) Since $\left(m_{s}\right)_{s \in S} \in N(\tilde{G})$, there is some $\left(m_{s}^{\prime}\right)_{s \in S} \in \tilde{\mu}\left(\left(m_{s}\right)_{s \in S}\right)$ such that $\left(m_{s}^{\prime}\right)_{s \in S} \in P(\tilde{G})$. Clearly, $\left(n_{s}+m_{s}^{\prime}\right)_{s \in S} \in \tilde{\mu}\left(\left(n_{s}+m_{s}\right)\right)$. By the induction hypothesis, $\left(n_{s}+m_{s}^{\prime}\right)_{s \in S} \in P(\tilde{G})$. Hence $\left(n_{s}+m_{s}\right)_{s \in S} \in N(\tilde{G})$.

Let us define an equivalence relation $\equiv$ on $\tilde{S}$ by that $\left(n_{s}\right)_{s \in S} \equiv$ $\left(m_{s}\right)_{s \in S}$ iff $n_{s} \equiv m_{s}(\bmod 2)$, for all $s \in S$. Then it is not difficult to see that $\equiv$ is a semicompatible relation on $\tilde{G}$. We define $L(G)=\tilde{G} / \equiv$. We denote the equivalence class of $\left(n_{s}\right)_{s \in S}$ by $\left[\left(n_{s}\right)_{s \in S}\right]$. Then we can define an operation $\bigoplus$ on $L(S)=\widetilde{S} / \equiv$ by $\left[\left(n_{s}\right)_{s \in S}\right] \oplus\left[\left(m_{s}\right)_{s \in S}\right]=\left[\left(n_{s}+m_{s}\right)_{s \in S}\right]$. Clearly, $L(S)$ is a vector space over $Z_{2}$ by this operation. By Lemma 3.3 , we have the following theorem.

Theorem 3.4. $P(L(G))$ is a subspace of $L(S)$.

Now, we define a mapping $f: S \rightarrow \widetilde{S}$ as follows: for any $t \in S, f(t)=$ 7) Here, $H(s) \oplus H(t)=(H(s)-H(t)) \cup(H(t)-H(s))$, i. e., the symmetric difference. 
$\left(n_{s}\right)_{s \in S}$, where $n_{t}=1$ and $n_{s}=0$ if $s \neq t$.

Clearly, $s \mapsto[f(s)]$ is an isomorphism into $G$ into $L(G)$. So, by identifying $s$ and $[f(s)]$, we may consider that $G \subset L(G)$. It is clear that $S$ is a basis of $L(S)$. So, any element $u \in L(S)$ can be written in the form $u=\sum_{s \in S} \oplus \alpha_{s}(u) s\left(\alpha_{s}(u) \in \mathbb{Z}_{2}\right)$. Now, for any $s \in S$ and $t \in \mu(s)$, we define a linear map $h_{s, t}$ on $L(S)$ by $h_{s, t}(u)=u \oplus \alpha_{s}(u)(s \oplus t)$. We set $H=\left\{h_{s, t} \mid s \in S, t \in \mu(s)\right\}$. Then it is easy to see that this $H$ satisfies the condition (II) of Definition 3.2. Therefore, $L(G)$ is a linear game.

Recall that $S$ is well-ordered by Lemma 1.2, i.e., $S=\left\{s^{0}, s^{1} s^{2}, \ldots\right\}$, where $s^{0}<s^{1}<s^{2}<s^{3}<\cdots$. Using this order $L(S)$ can be also well-ordered as follows: Let $s=\sum_{\oplus} \alpha_{i} s^{i}$ and $t=\sum_{\oplus} \beta_{i} s^{i}$ be two elements in $L(S)$, where $\alpha_{i}, \beta_{i} \in \mathbb{Z}_{2}$. Then we define $s \leqq t$ iff $\sum \alpha_{i} 2^{i} \leqq \sum \beta_{i} 2^{i}$.

It can be easily seen that this order has the following property:

For any $s \in L(S)$ and $h \in H(s), h(s)<s$.

Lemma 3.5. For any nonzero $s \in L(S)$, let $K(s)$ be the greatest integer $k$ such that $\alpha_{s^{k}}(s)=1$. Let $F=S-\left\{s^{k} \mid k=K(s)\right.$ for some $\left.s \in P(L(G))\right\}$. Let $Q$ be the subspace of $L(S)$ spanned by $F$. Then $L(S)=P(L(G)) \oplus Q$, the direct sum of two vector subspaces.

The proof is immediate from the definition of $F$.

Since $L(S)=P(L(G)) \oplus Q$, a linear map $q: L(S) \rightarrow Q$ can be defined as the projection from $L(S)$ to $Q$. Then the following lemma is also immediate.

Lemma 3.6. Let []$: L(S) \rightarrow L(S) / P(L(G))$ be the canonical mapping. Then for any $s \in L(S), q(s)=\min [s]$.

Lemma 3.7. For any $s \in L(S)$ and $t \in Q$ such that $t<q(s)$, there is some $h \in H(s)$ such that $q(h(s))=t$.

Proof. (1) First we show $s \oplus t \notin P(L(G))$. Suppose that $s \oplus t \in P(L(G))$, then $0=q(s \oplus t)=q(s) \oplus q(t)=q(s) \oplus t$. Hence $t=q(s)$. A contradiction.

(2) Since $s \oplus t$ is unsafe, there is some $h \in H(s \oplus t)$ such that $h(s \oplus t) \in$ $P(L(G))$. Hence $0=q(h(s)) \oplus q(h(t))$. That is, $q(h(s))=q(h(t))$, where 
either $h \in H(s)-H(t)$ or $h \in H(t)-H(s)$. Suppose $h \in H(t)-H(s)$. Then $h(s)=s$. Hence $q(h(t))=q(s)>t>h(t) \geqq q(h(t))$. This is a contradiction. So, $h \in H(s)-H(t)$. Thus we know $h \in H(s)$ and $q(h(s))=t$.

Lemma 3.8. For any $s \in L(S)$ and $h \in H(s), q(s) \neq q(h(s))$.

Proof. Since $s=q(s) \oplus(q(s) \oplus s)$, either (1) $h \in H(q(s))-H(q(s) \oplus s)$ or (2) $h \in H(q(s) \oplus s)-H(q(s))$.

(Case 1) $h(s)=h(q(s)) \oplus(q(s) \oplus s)$. Hence $q(h(s))=q(h(q(s)))$. By Lemma 3.6, we have $q(h(q(s)))<q(s)$. Hence $q(s) \neq q(h(s))$.

(Case 2) In this case $h \in H(s)-H(q(s))$. Hence $q(h(q(s) \oplus s))=q(h(q(s)) \oplus h(s))=q(q(s) \oplus h(s))=q(s) \oplus q(h(s))$. But, since $q(s) \oplus s$ is safe, $q(h(q(s) \oplus s))) \neq 0$. Thus $q(s) \neq q(h(s))$.

Now, let $D=\{n \in N \mid n<\overline{\bar{Q}}\} \oplus$, where $\overline{\bar{Q}}$ denotes the cardinality of the set $Q$. If we denote the binary sum of integers by $\oplus$, then $D$ is a vector space over $\boldsymbol{Z}_{2}$ with respect to this $\oplus$. We denote the $i$ th least element of $F^{8)}$ by $f^{i-1}$, then any $s \in Q$ is of the form $s=\sum_{\oplus} \alpha_{i} f^{i}\left(\alpha_{i} \in Z_{2}\right)$.

We define a mapping $\varphi: Q \rightarrow D$ by $\varphi\left(\sum_{\oplus} \alpha_{i} f^{i}\right)=\sum_{\oplus} \alpha_{i} 2^{i}$. Then we can easily verify that $\varphi$ is an onto isomorphism with respect to $\oplus$ and $\leqq$. Hence, by Lemmas 3.7 and 3.8 , we see that $\varphi \circ q$ is the Grundy function on $L(G)$. Thus we obtain the following theorem.

Theorem 3.9. The Grudy function on $L(G)$ is a linear map from $L(G)$ to $\mathbb{N}$.

Definition 3.10. A game $G=(S, \mu)$ is called the direct sum of $n$ games $G_{1}=\left(S_{1}, \mu_{1}\right), \ldots, G_{n}=\left(S_{n}, \mu_{n}\right)$ if

(1) $S=S_{1}+S_{2}+\cdots+S_{n}$ (direct sum of sets) and

(2) $\mu(s)=\mu_{i}(s)$, where $s \in S_{i}$.

We denote this game by $G_{1}+\cdots+G_{n}$.

Definition 3.11. A game $G=(S, \mu)$ is called the Cartesian product of $n$ games $G_{1}=\left(S_{1}, \mu_{1}\right), \ldots, G_{n}=\left(S_{n}, \mu_{n}\right)$ if

(1) $S=S_{1} \times \cdots \times S_{n}$ and

8) See Lemma 3.5. 
(2) $\mu(s)=\bigcup_{i=1}^{n}\left\{s_{1}\right\} \times \cdots \times\left\{s_{i-1}\right\} \times \mu_{i}\left(s_{i}\right) \times\left\{s_{i+1}\right\} \times \cdots \times\left\{s_{n}\right\}$, where $s=\left(s_{1}, \ldots, s_{n}\right)$.

We denote this game by $G_{1} \times \cdots \times G_{n}$.

Now, let $G_{i}=\left(S_{i}, \mu_{i}\right)(i=1, \ldots, n)$ be any games. We define a mapping $\psi: S_{1} \times \cdots \times S_{n} \rightarrow L\left(S_{1}+\cdots+S_{n}\right)$ by $\phi\left(s_{1}, \cdots, s_{n}\right)=s_{1} \oplus \cdots \oplus s_{n}$. (Recall that we consider that $S_{i}\left(S_{1}+\cdots+S_{n}\left(L\left(S_{1}+\cdots+S_{n}\right)\right.\right.$.) Then it is clear that this $\psi$ is an isomorphism from $G_{1} \times \cdots \times G_{n}$ to $L\left(G_{1}+\cdots+G_{n}\right)$. Thus, as a corollary of Theorem 3.9, we obtain Grundy's theorem.

Theorem 3.12. (Grundy) Let $G=G_{1} \times \cdots \times G_{n}$ be the Cartesian product of $n$ games. Then for any $s=\left(s_{1}, \cdots, s_{n}\right) \in S_{1} \times \cdots \times S_{n}$,

$$
g(s)=g\left(s_{1}\right) \oplus \cdots \oplus g\left(s_{n}\right) \text {. }
$$

\section{§4. Linear Games}

Throughout this $\S$ we fix a linear game $G=(S, \mu)$. And we suppose $H$ is a subset of $\operatorname{Map}(S, S)$ which satisfies the condition (II) of Definition 3.2 .

By the condition (II) (a) of Definition 3.2, the set $T$ of all terminals of $G$ forms a subspace of $S$. And the equivalence relation induced by $T$ (i.e. $s T t$ iff $s \oplus t \in T$ ) is a compatible relation on $G$. Then it is clear that the quotient game $G / T$ is also a linear game and has only one terminal 0. Remark that this also means that if $H(s)=H(t)$ then $s=t$. Our aim in this $\S$ is to show that $g_{G}$ is a linear map. (See Theorem 4.5.) To this end we have only to show that $g_{G / T}$ is linear since if we denote the canonical mapping from $S$ to $S / T$ by $\pi$ then $g_{G}=g_{G / T} \circ \pi$. So, in this $\S$, we assume $G$ has only one terminal 0 .

Definition 4.1. An element $s \in S$ is called simple if for any $t \in S$ either $H(s) \subset H(t)$ or $H(s) \cap H(t)=\emptyset$.

An element $s \in S$ is called complicated if for any simple $t$, $H(s) \cap H(t)=\varnothing$.

It can be easily verified that the set $C$ of all the complicated ele- 
ments is a subspace of $S$. Let $M$ be the subspace of $S$ spanned by all simple elements.

Lemma 4.2. $S=M \oplus C$.

Proof. (1) Suppose $S \in M \cap C$. Then $H(s) \cap H(s)=\varnothing$. Hence $s=0$, that is, $M \cap C=\{0\}$.

(2) Take any $s \in S$. Let $V=\{v \in S \mid H(s) \supset H(v), \quad v$ is simple $\}$. Then $V$ is finite since $H(s)$ is finite and $H(v)=H\left(v^{\prime}\right)$ implies $v=v^{\prime}$. Let $t=\sum_{v \in V} \oplus v$. Then $H(t)=\bigcup_{v \in V} H(v)$ (disjoint union). Let $u=s \oplus t$. Then, since $H(s) \supset H(t), H(u)=H(s)-H(t)$. If we suppose $u$ is not complicated, then there is a simple $w$ such that $H(u) \cap H(w) \neq \varnothing$. It follows that $H(s) \cap H(w) \neq \emptyset$. Since $w$ is simple, this means $H(s) \supset H(w)$. Hence $w \in V$. Since $H(t)=\bigcup_{v \in V} H(v), H(t) \supset H(w)$. Then $H(u) \cap H(t) \supset H(u) \cap H(w) \neq \varnothing$. This contradicts the fact that $H(u)=H(s)-H(t)$. Therefore $u \in C$. Thus $s=t \oplus u$ for some $t \in M$ and $u \in C$.

Lemma 4.3. $C=\{0\}$.

Proof. Suppose $C \neq\{0\}$. Then $S-M \neq \varnothing$. Since $G$ is progressively finite, there is some $s_{1} \in S-M$ such that $\mu\left(s_{1}\right) \subset M$. Let $s_{1}=m_{1} \oplus c_{1}$, where $m_{1} \in M$ and $c_{1} \in C$. Then $H\left(s_{1}\right)=H\left(m_{1}\right) \cup H\left(c_{1}\right)$ (disjoint). Let $h$ be any element in $H\left(c_{1}\right)$. Then $h \in H\left(s_{1}\right)$. Hence, $h\left(c_{1}\right)=h\left(s_{1} \oplus m_{1}\right)=h\left(s_{1}\right) \oplus m_{1} \in M$. Thus we have $\mu\left(c_{1}\right) \subset M$. By Lemma $4.2, c_{1}$ is not simple. So, there exists $t \in S$ such that $H\left(c_{1}\right) \not \subset H(t)$ and $H\left(c_{1}\right) \cap H(t) \neq \varnothing$. Let $t=m_{2} \oplus c_{2}$, where $m_{2} \in M$ and $c_{2} \in C$. Then $H\left(c_{1}\right) \not \subset H\left(c_{2}\right)$ and $H\left(c_{1}\right) \cap H\left(c_{2}\right) \neq \varnothing$. Take $h \in H\left(c_{1}\right) \cap H\left(c_{2}\right)$, and let $s=h\left(c_{1}\right)(\in M)$. Then $h\left(c_{2}\right)=s \oplus c_{1} \oplus c_{2}$, since $h \notin H\left(c_{1} \oplus c_{2}\right)$. Next, take $h^{\prime} \in H\left(c_{1}\right)-H\left(c_{2}\right)$, and let $s^{\prime}=h^{\prime}\left(c_{1}\right)$ $(\in M)$. Then $h^{\prime}\left(c_{1} \oplus c_{2}\right)=s^{\prime} \oplus c_{2}$. Hence, we obtain the following loop.

$$
c_{2} \mid \underset{h}{\longrightarrow} \oplus \oplus c_{1} \oplus c_{2} \overrightarrow{h^{\prime}} s \oplus s^{\prime} \oplus c_{2} \underset{h}{\longrightarrow} s^{\prime} \oplus c_{1} \oplus c_{2} \overrightarrow{h^{\prime}} c_{2}
$$

This contradicts that $G$ is progressively finite.

Lemma 4.4. The set $B$ of all the nonzero simple elements is a basis 
of $S$.

Proof. By Lemmas 4.2 and $4.3, B$ spans $S$. We now show that $B$ is linearly independent. Let $\left\{s_{1}, \ldots, s_{k}\right\} \subset B$, and suppose $\sum_{i=1}^{k} \oplus_{i}=0$. Then $H\left(\sum_{i=1}^{k} \oplus s_{i}\right)=\bigcup_{i=1}^{k} H\left(s_{i}\right)=\varnothing$. Hence $H\left(s_{i}\right)=\varnothing$ for any $i$, that is, $s_{i}=0$ for any $i$.

For any $s, t \in S$, we say $s$ appears in $t$ if $s$ is simple and $H(s) \subset H(t)$. Then, as we have seen in Lemma $4.2, t$ is the sum of simple elements appearing in $t$.

The following theorem is an extension of Theorem 3.9 .

Theorem 4.5. The Grundy function on any linear game is a linear map.

Proof. In $\S 3$, we defined an isomorphism from $G$ to $L(G)$. (See the discussion which follows Theorem 3.4). We denote this isomorphism by $\varphi$. We define a linear map $\psi: L(S) \rightarrow S$ as follows: Any $s \in L(S)$ is of the form $s=\varphi\left(s_{1}\right) \oplus \cdots \oplus \varphi\left(s_{k}\right)$, where $s_{1}, \cdots, s_{k}$ are in $S$. We define $\phi(s)=$ $s_{1} \oplus \cdots \oplus s_{k}$. Then $\psi$ is well-defined since $\varphi(s)$ is a basis of $L(S)$.

Let us define an equivalence relation $\equiv$ on $L(S)$ by the condition that $s \equiv t$ iff $\phi(s)=\psi(t)$. We now show that $\equiv$ is a semicompatible relation on $L(G)$. Suppose $s=\varphi\left(s_{1}\right) \oplus \cdots \oplus \varphi\left(s_{m}\right)$ and $t=\varphi\left(t_{1}\right) \oplus \cdots \oplus \varphi\left(t_{n}\right)$ are in $L(S)$, and $\phi(s)=\psi(t)$. Take any $h \in H(s)$. Then $h \in H\left(\varphi\left(s_{i}\right)\right)$ for a unique $s_{i}$. Since $\varphi$ is an isomorphism, there is a unique $\bar{h} \in H\left(s_{i}\right)$ such that $h\left(\varphi\left(s_{i}\right)\right)=\varphi\left(\bar{h}\left(s_{i}\right)\right)$. For this $\vec{h}$ there is a unique simple $u \in S$ such that $\bar{h} \in H(u)$. Clearly, $u$ appears in $s_{i}$. Hence $h(s)=s \oplus \varphi\left(s_{i}\right) \oplus h\left(\varphi\left(s_{i}\right)\right)=$ $s \oplus \varphi\left(s_{i}\right) \oplus \varphi\left(\bar{h}\left(s_{i}\right)\right)=s \oplus \varphi\left(s_{i}\right) \oplus \varphi\left(s_{i} \oplus u \oplus \bar{h}(u)\right)$. Thus $\psi(h(s))=\psi(s) \oplus u \oplus \bar{h}(u)$. Here we have two cases.

(Case 1) The case where $u$ appears in some $t_{j}(j=1, \ldots, n)$ : In this case, we can show, similarly as above, that $\phi(h(t))=\phi(t) \oplus u \oplus \vec{h}(u)$. Since $\psi(s)=\psi(t)$, we have $\psi(h(s))=\psi(h(t))$.

(Case 2) Otherwise $u$ does not appear in any $t_{j}(j=1, \ldots, n)$. Since $\psi(s)=\psi(t)$, this means that $u$ appears even number of times in $S_{j}$ 's $(j=1, \ldots, m)$. Hence $u$ must appear in some $s_{k}$ other than $s_{i}$. Then there 
exists $h^{\prime} \in H\left(\varphi\left(s_{k}\right)\right)$ such that $h^{\prime}\left(\varphi\left(s_{k}\right)\right)=\varphi\left(\bar{h}\left(s_{k}\right)\right)$. It is easy to see that $h^{\prime}$ is also in $H(h(s))$. So, $h^{\prime}(h(s))=s \oplus \varphi\left(s_{i}\right) \oplus \varphi\left(s_{i} \oplus u \oplus \bar{h}(u)\right) \oplus \varphi\left(s_{k}\right) \oplus \varphi$ $\left(s_{k} \oplus u \oplus \vec{h}(u)\right)$. Hence $\phi\left(h^{\prime}(h(s))\right)=\psi(s)$.

The results of both cases mean that $\equiv$ is a semicompatible relation on $G$. Now, we can show that $g_{G}$ is linear. Let $s$ and $t$ be any elements in $S$. Then, since $\varphi$ is an isomorphism, $g_{G}(s \oplus t)=g_{L(G)}(\varphi(s \oplus t))$. Obviously, $\varphi(s \oplus t) \equiv \varphi(s) \oplus \varphi(t)$. Hence, by Lemma 2.4 and Theorem 3.9, $g_{L(G)}(\varphi(s \oplus t))=g_{L(G)}(\varphi(s) \oplus \varphi(t))=g_{L(G)}(\varphi(s)) \oplus g_{L(G)}(\varphi(t))=g_{G}(s) \oplus g_{G}(t)$. This completes our proof.

Corollary 4.6. $P(G)$ is a subspace of $S$.

\section{References}

[1] Banerji, R. B., Theory of Problem Solving, Elsevier, New York, London, Amsterdam, 1968.

[2] Berge, C., The Theory of Graphs, Methuen, London, Wiley, New York, 1966.

[3] Grundy, P.M. and C.A.B. Smith. Disjunctive games with the last player loosing, Proc. Cambridge Philos. Soc. 52 (1956), 527-533.

[4] Holladay, J.C., Cartesian products of termination games, Contributions to the Theory of Games, vol. III, Princeton University Press, Princeton (1957) 189-200.

[5] Hitotumatu, S. Mathematical Theory of Nim-type Games (in Japanese), Morikita, Tokyo, 1968. 